\title{
Immunosuppressive Acidic Protein- Haematological correlates in HIV infected subjects
}

\author{
Olayanju Ayodeji Olusola', Oyebola Temiloluwa David², Akele Richard Yomi², Enitan Seyi \\ Samson ${ }^{4}$, Oladele Ayowole Abraham ${ }^{5}$, Sanya Joseph Olurotimi ${ }^{6}$, Olayanju Aimien Joy ${ }^{7}$ \\ ${ }^{1}$ Senior Lecturer, Department of Medical Laboratory Science, College of Medicine and Health Sciences, AfeBabalola \\ University, PMB 5454, Ado-Ekiti, Ekiti State, Nigeria, ${ }^{2}$ Research Student, ${ }^{3}$ Senior Lecturer, Department of Medical \\ Laboratory Science, College of Medicine and Health Sciences, AfeBabalola University, PMB 5454, Ado-Ekiti, Ekiti \\ State, Nigeria, ${ }^{4}$ Lecturer, Department of Medical Laboratory Science, Babcock University, PMB 21244, Ilishan-Remo, \\ Ogun State, Nigeria, ${ }^{5}$ Reader, ${ }^{6}$ Professor, ${ }^{7}$ Instructor, Department of Medical Laboratory Science, College of Medicine \\ and Health Sciences, AfeBabalola University, PMB 5454, Ado-Ekiti, Ekiti State, Nigeria
}

A B S T R A C T

Background: Immunosuppressive Acidic protein (IAP) is a marker of the extent of immune defects occurring in most cancers. Its correlation with CD4 cell count used as an indicator of immune function and disease progression in Human Immuno-deficiency Virus (HIV) infection is not well documented. Aims and Objectives: To determine if IAP levels correlates with immunosupression and haematopathology occurring in HIV/AIDS infection. Materials and Methods: This cross sectional study was conducted at the Federal Teaching Hospital, Ido-Ekiti. One hundred and five participants consisting of 85 HIV infected test subjects and 20 control subjects were enrolled into the study. CD4 counts was obtained using SL Cyflow machine, IAP levels determined using ELISA kit for human IAP and Full blood count for all participants was obtained using Sysmex KX-21N Haematology Analyzer. Regression and correlation analysis was done on data using SPSS 28. Results: IAP showed a negative correlation with CD4 count $(r=-0.6),(r=-0.9)$ and $(r=-0.2)$ in the ART, NART and control groups. The pattern of the results was similar with other parameters except in Neutrophils $(r=0.2)(r=0.3)$ and (0.1), Eosinophil $(r=0.6)(r=-0.4)$ and $(r=-0.2)$ and Lymphocyte $(r=-0.3)(r=-0.02)$ and $(r=0.05)$ in the ART, NART and control groups respectively. Conclusion: The outcome of this study show that a strong negative relationship exist between IAP and other immunohaematological parameters used for monitoring Immune status in HIV infection; however the information gotten is not sufficient to indicate IAP as a predictor of immune status in HIV infection. Further studies are therefore required to better elucidate the mechanism of increased IAP levels at different clinical stages of HIV infection.

Key words: Immunosuppressive acidic protein, CD4, HIV/AIDS, Immunosuppression, Haematopathology

\section{INTRODUCTION}

The Acquired Immunodeficiency Syndrome (AIDS) is due to infection by the Human Immune-deficiency Viruses (HIV). The infections cause progressive destruction of the Cell Mediated Immune (CMI) system, primarily by eliminating CD4+ T-helper lymphocytes. Opportunistic infections and tumors follow causing a progressive damage of the body's immune system. ${ }^{1,2}$

The damage done to the immune system results in immunological and haematological complications causing a number of opportunistic infections (OIs). Haematological complications have been documented to be the second most 
common cause of morbidity and mortality in HIV patients and are generally marked with cytopenia such as anaemia, neutropenia, lymphopenia and thrombocytopenia. ${ }^{3,4}$

The incidence and severity of the cytopenia generally correlate to the stage of the disease with anaemia being the most commonly encountered haematologic abnormality and a significant predictor of progression to AIDS or death. ${ }^{5}$

The progression of the HIV infection may be measured through various outcome measures with measurement of CD4 count as a gold standard. ${ }^{6}$ Obtaining CD4 counts requires the use of expensive equipment, which are not readily available in resource-limited settings in Nigeria. The identification of a surrogate laboratory tests that can help the clinician to predict progression as well as CD4 cell count does is useful not only to monitor the patients' disease evolution but also to define the appropriate time to initiate treatment. ${ }^{7,8}$

Immunosupressive Acidic Protein (IAP) is an acute-phase reactant which has a close correlation with the impairment of the hosts immunity. It has been found to suppress both phytohemagglutinin-induced lymphocyte blast formation and mixed lymphocyte reaction in vitro. ${ }^{9}$ It is a glycoprotein containing $31.5 \%$ Carbohydrates with a molecular weight of 50,000 and a single iso-electric point of 3.0. Impairment of host immunity has been reported in a variety of cancer patients, especially in advanced stage of the disease and it has been demonstrated that IAP is the main component of $\alpha-A G$ which causes these Impairment of host immunity seen in different cancers. ${ }^{10}$

IAP is synthesized in the liver and has a serum concentration that increases in response to tissue injury, inflammation or infection. An increase in its concentration has been implicated in the immunosuppresion in tumor bearing animals and humans although the mechanism by which these occurs is not clearly stated. ${ }^{11,12}$ Due to the inflammation or infection in HIV infection, It is highly plausible that there is correlation between IAP levels Immuno-haematopathology occurring in HIV infection. Based on the fact that IAP levels are easily estimated in human serum with a cost effective procedure using ELISA, there is the need to investigate if such relationship exists as this will provide a surrogate to the use of the more expensive CD4 count as a marker for the disease progression. ${ }^{13}$

\section{MATERIALS AND METHODS}

\section{Study area}

This was a cross sectional study conducted at the HIV care and support center of the Federal Teaching Hospital, Ido in Ekiti State which caters for Ido and the surrounding Towns like Ifaki, Otun, Aiyee in Ekiti-State.

\section{Subjects}

A total of 105 randomly selected participants who met the inclusion criteria of the study were enrolled for this study. Eighty-five of the participants were HIV positive individuals of the HIV care and support center of the Federal Teaching Hospital, Ido and 20 were HIV negative control subjects.

\section{Sample size determination}

A minimum sample size that is representative of the study population was determined using a standardized method:

$\mathrm{r}+1 \mathrm{SD}^{2}(\mathrm{Z}+\mathrm{Z})^{2} \quad \mathrm{r} \quad \mathrm{d}^{2}$

$\mathrm{SD}$ is taken to be 0.8 from previous study ${ }^{14}$

$r$ is ratio of control to cases $(1: 5)=0.2$,

$\mathrm{Z}$ is standard normal variate for power $80 \%=0.84$,

$\mathrm{Z}$ is standard normal variate for $0.05 \%$ tolerable error $=$ 1.96 ,

$\mathrm{d}$ is the expected mean difference between case and control taken as $600 \mathrm{cell} / \mu \mathrm{l}$.

$0.2+10.8^{2}(0.84+1.96)^{2}$

$0.2600^{2}$

$=105$ total participants

(For every 5 HIV infected subjects there is 1 control subject)

The sample size was calculated to be 105 having 85 test subjects and 20 control individuals.

\section{Ethical approval}

In line with Helsinki Declaration, approval for this study was obtained from the Health Research Ethics Committee of the Federal Teaching Hospital., IdoEkiti, Ekiti State.

\section{Inclusion criteria}

1. May or may not be undergoing Anti Retroviral therapy.

2. Subjects should be at varying stages of disease progression.

3. Should give his or her informed consent on the research study.

4. Subjects that are not HIV positive

\section{Exclusion criteria}

1. Subjects that are not HIV positive except control participants.

2. Subjects who do not give their informed consent. 
Informed consent

The participants were informed on the objectives, benefits and procedure of the study and they were assured of confidentiality, voluntariness and protection. A written Informed consent was obtained from each of the participant before enrolment into the study. They were also made aware of their option to withdraw from the study any time without losing any of the benefits and healthcare given by the hospital. The investigation was carried out at no cost to the participants.

\section{Research design}

Venous blood was collected from them and analyzed for CD4 count, complete blood count and IAP concentration. Results obtained will be tabulated and analyzed using appropriate statistical tool.

\section{Sample collection}

5 milliliters $(5 \mathrm{mls})$ of venous blood was collected from the antecubital fossa using aseptic technique.

\section{Sample analysis}

Full blood count and CD4 count were estimated and plasma separated for IAP concentration estimation, samples were analyzed for Full Blood Count and CD4 count within 6 hours of collection using Sysmex KX-21N Haematology and SL Cyflow machine. IAP was determined using the Enzyme Linked Immunosorbent Assay (ELISA) machine.

\section{Statistical analysis}

All experimental results were first evaluated to establish the necessity for using parametric statistics. The data was then determined to have a normal distribution. Degree of correlation of each parameter analyzed with IAP was then determined using Statistical Packages for Social Science (SPSS) version 28.

\section{RESULTS}

The results of this study was presented using scatter plot graphs (Figures 1-9) showing correlation relationship between Immunosuppressive Acidic Protein (IAP) and other variables estimated in the study. IAP did not have any correlation with percentage Monocyte and percentage Basophil differential count. The (r) values each of the ART, NART and control group in all nine parameter with correlation IAP levels in the study are also stated.IAP had negative correlation with CD4 count, TWBC and the RBC dependent parameters.

\section{DISCUSSION}

The Human Immunodeficiency Virus (HIV) epidemic has spawned a scientific effort unprecedented in the history of infectious disease research. ${ }^{1}$ The need to find a surrogate to expensive CD4 cell count for laboratory monitoring and management of HIV has been further emphasized by the reduction in support funds for the management of HIV infected people in resource poor settings in Africa. ${ }^{15}$ This study was designed to assess the Haematological correlates of Immunosupressive Acidic Protein marker (IAP) in HIV infection and the possibility of using IAP as a surrogate marker for CD4 cell count in the monitoring of immune status in HIV infected individuals. IAP is an acute-phase reactant and IAP is a glycoprotein containing $31.5 \%$ carbohydrates with a molecular weight of 50,000 and a single isoelectric point of 3.0. The presence of an unusual amount of immunosuppressive proteins in the serum of cancer patients has been suggested as one of the factors which lead to depression of host immunity. ${ }^{10}$

In this study, there was a negative correlation between the levels of CD4, TWBC and IAP, in the ART, NART and in the control group as shown in Figure 1 and Figure 2, respectively. The result correlates with the findings in other immunosuppressive diseases as there is paucity of information on IAP in relations to HIV. The immunosuppressive disease conditions include lung cancer as explained by study conducted elsewhere. ${ }^{10} \mathrm{~A}$ similar result was obtained in Primary cervical cancer patients ${ }^{16}$ and also in renal cancer. ${ }^{17,18}$ It is expected that there should be a relationship between the IAP levels in HIV infection and all these diseases as they are characterized by suppressed immune function, tissue injury, inflammation and infection as seen in HIV infection. IAP increases with reducing CD4 count and TWBC (increasing disease severity) probably due to its increased synthesis in the liver in response to tissue injury, inflammation or infection which is typical of immunosuppressive states and advancing HIV infection. ${ }^{11}$

This study also found a positive correlation in the ART group between red blood cell, packed cell volume, haemoglobin and percentage eosinophil with the levels of IAP as shown in Figure 3, Figure 4, Figure 5 and Figure 9, respectively. This finding was inconsistent with those from similar studies. ${ }^{3,19}$ The inconsistency in the ART group with the previous study is possibly due to a better adherence to the treatment regimen by the patients involved in our study. However, there was a negative correlation in the NART group. This is in agreement earlier reported studies ${ }^{3,20}$ possibly due to the absence of therapy and thus a full effect of HIV infection on haematopoiesis as seen in anaemia of chronic disease affecting the kidney's production of erythropoietin. The control group also showed a negative correlation which is in agreement with earlier conducted studies. ${ }^{3,19}$ This is due to the fact that IAP synthesis in the liver increases in response to tissue injury, infection or inflammation which are not observed in HIV infection 


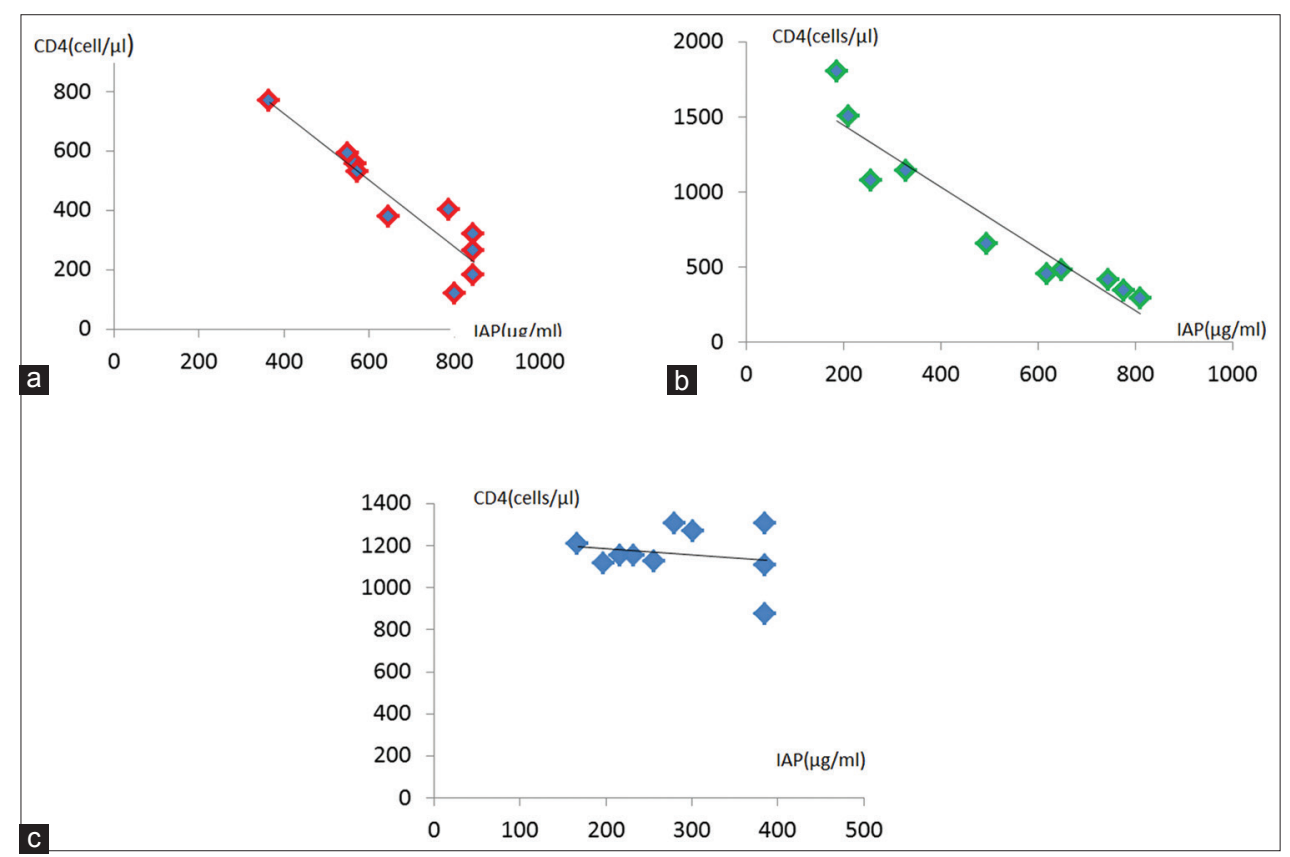

Figure 1: Scatter Plot showing relationship between IAP and CD4+ cell count in (a) ART (b) NART and (c) control group. (a) There is a negative correlation between the levels of IAP and CD4 in this group $(r=-0.6)$. (b) There is a very strong negative correlation between the levels of IAP and CD4 in this group ( $r=-0.9)$, (c) There is a very weak negative correlation between the levels of IAP and CD4 in this group ( $r=-0.2)$

KEY

- ART - HIV subjects on Anti Retroviral therapy

- NART - HIV subjects not on Anti Retroviral therapy

- IAP - Immunosuppresive Acidic Protein

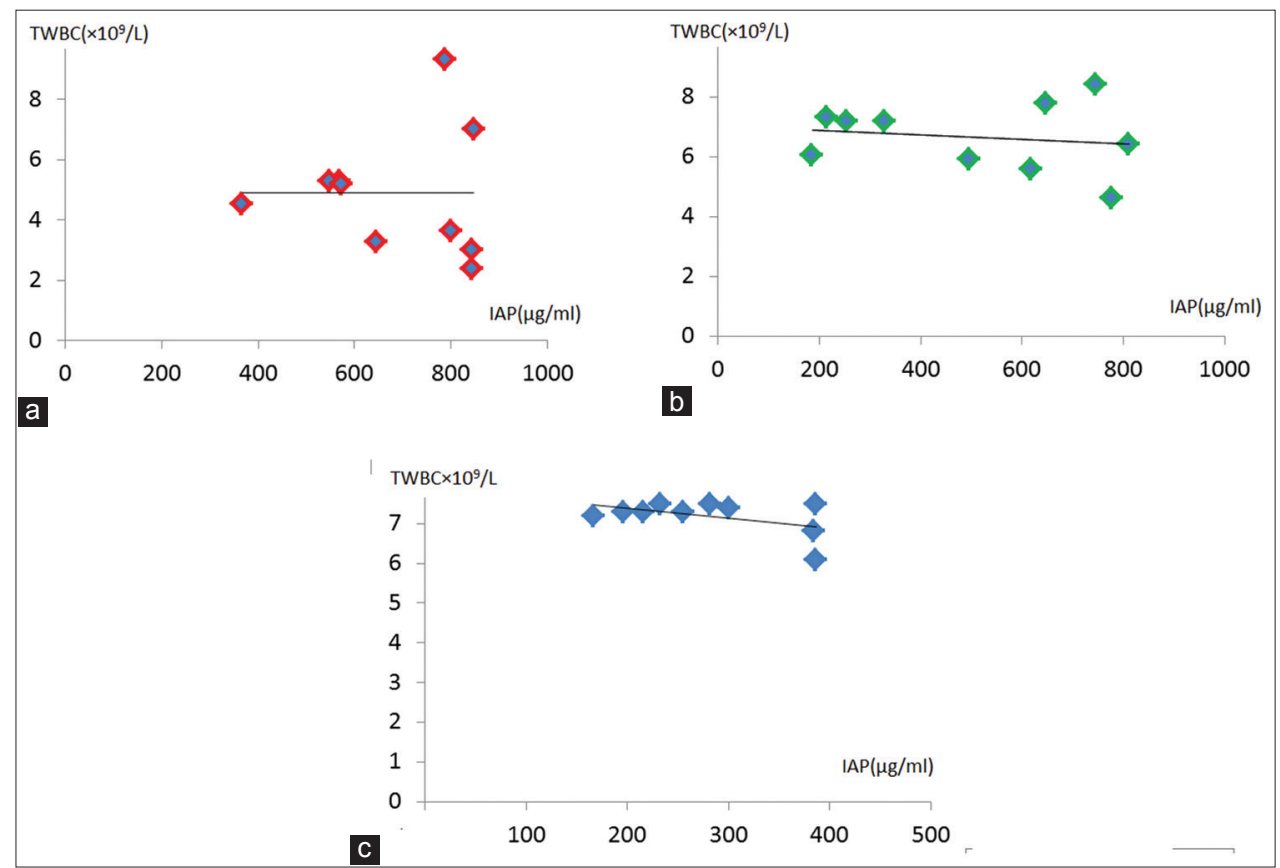

Figure 2: Scatter Plot showing relationship between IAP and TWBC count in (a) ART (b) NART and (c) control group, (a) There is a negative correlation between the levels of IAP and TWBC in this group ( $r=-0.7)$. (b) There is negative correlation between the levels of IAP and TWBC in this group $(r=-0.5)$. (c)There is negative correlation between the levels of IAP and TWBC in this group $(r=-0.3)$

KEY

- TWBC - Total White Blood Cell 


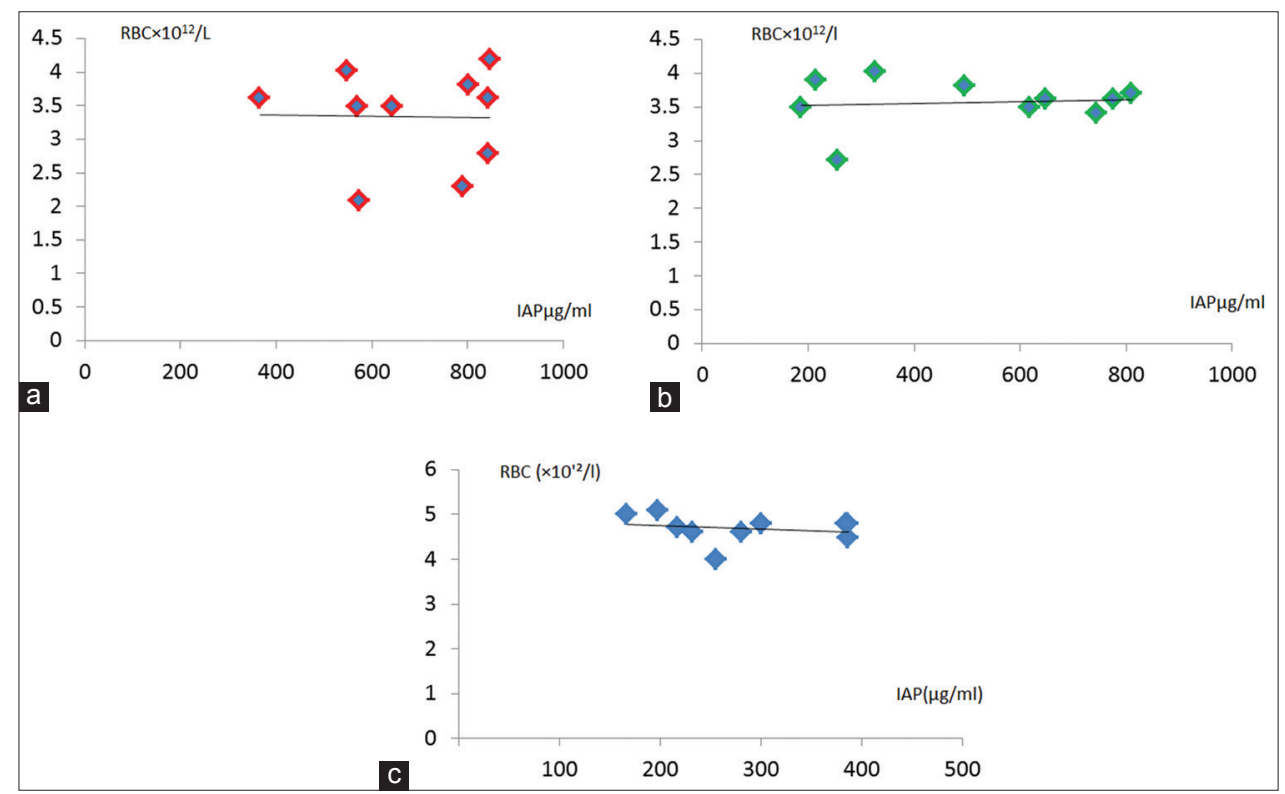

Figure 3: Scatter Plot showing relationship between IAP and RBC count in (a) ART (b) NART and (c) control group, (a) There is a very weak positive correlation between the levels of IAP and RBC in this group $(r=0.01)$. (b) There is a negative correlation between the levels of IAP and $R B C$ in this group $(r=-0.6)$. (c) There is a negative correlation between the levels of IAP and RBC in this group $(r=-0.3)$

KEY

- RBC - Red Blood Cell

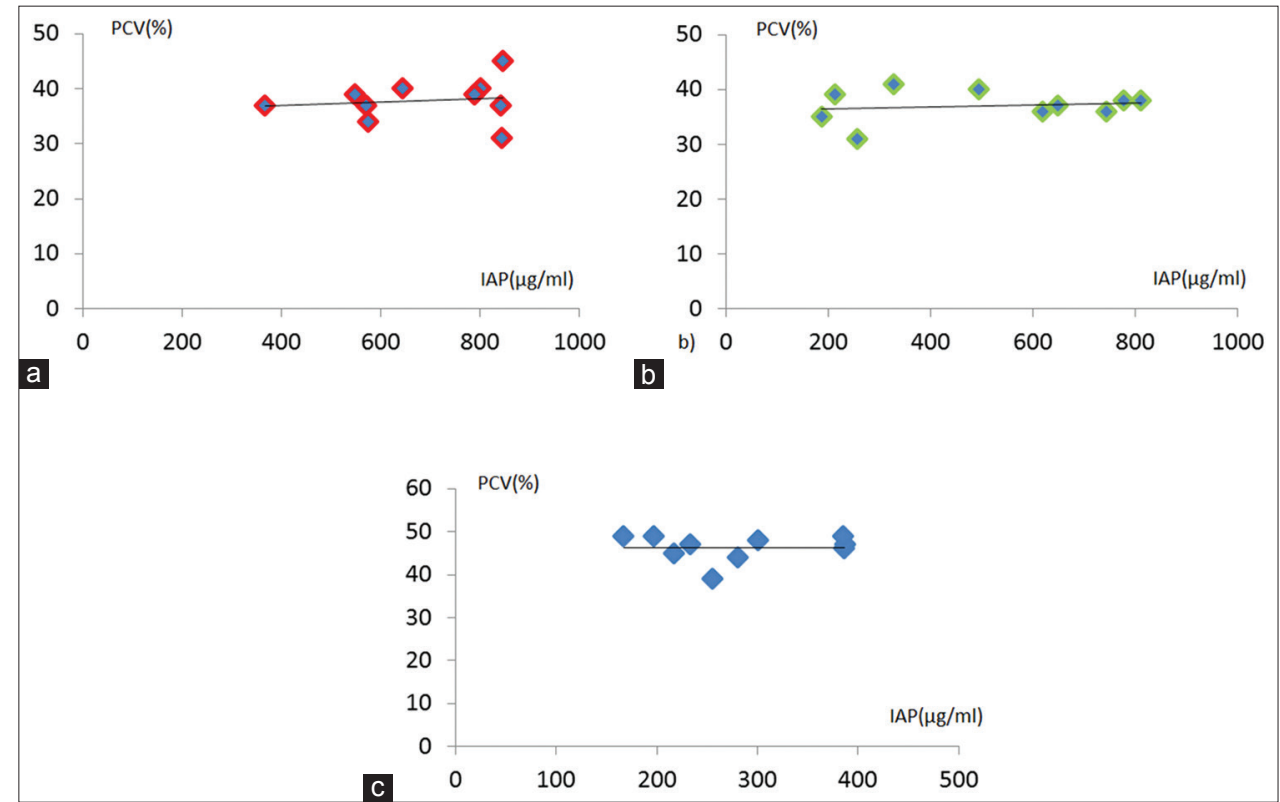

Figure 4: Scatter Plot showing relationship between IAP and PCV in (a) ART (b) NART and (c) control group. (a) There is a very weak positive correlation between the levels of IAP and PCV in this group $(r=0.09)$. (b) There is a negative correlation between the levels of IAP and PCV in this group $(r=-0.5)$. (c) There is a negative correlation between the levels of IAP and PCV in this group $(r=-0.2)$

KEY

- PCV - Packed Cell Volume

or immunosuppressive conditions alone but also in some parasitic or inflammatory conditions which may happen to cause premature destruction of red blood cells as seen in Malaria parasite infection or increased turnover of eosinophil.
This study also found that reticulocyte count had a negative correlation in the ART group and NART with the levels of IAP and a positive correlation in the control group as seen in Figure 6. This is in good agreement with earlier studies $^{21,22}$ possibly because of the anaemia of chronic 


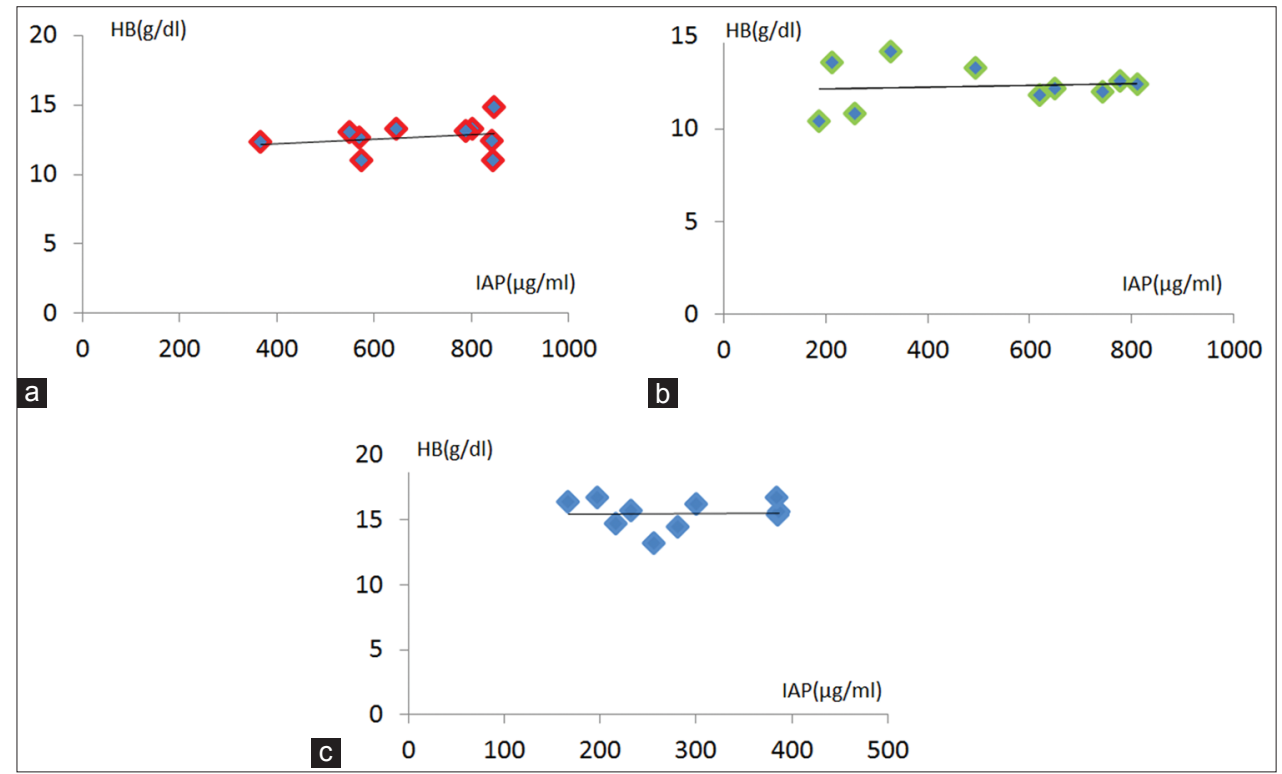

Figure 5: Scatter Plot showing relationship between IAP and Haemoglobin Concentration in (a) ART (b) NART and (c) control group, (a) There is a Positive correlation between the levels of IAP and HB in this group $(r=0.2)$. (b) There is a Negative correlation between the levels of IAP and HB in this group $(r=-0.5)$. (c) There is a Negative correlation between the levels of IAP and HB in this group $(r=-0.2)$

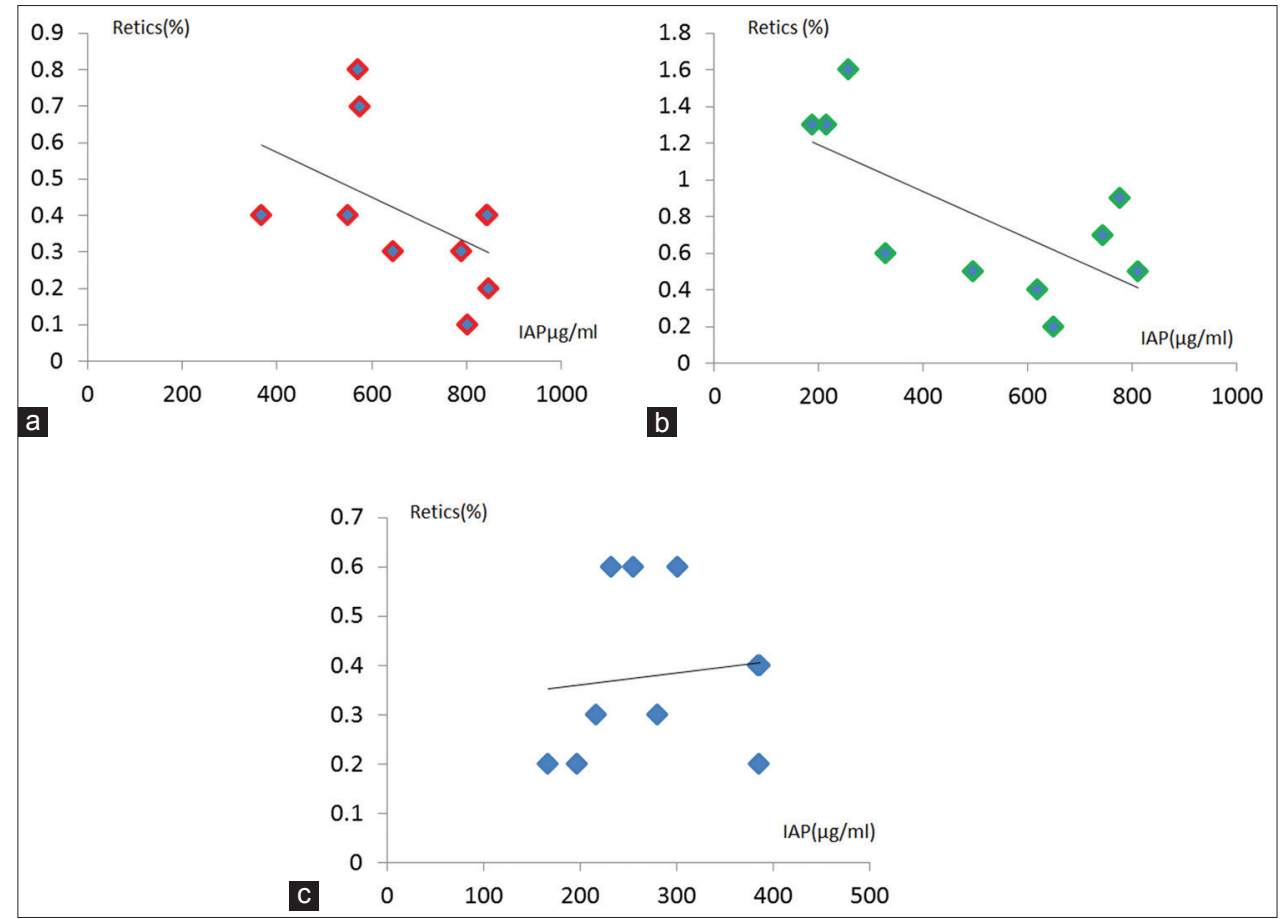

Figure 6: Scatter Plot showing relationship between IAP and Reticulocyte Count in (a) ART (b) NART and (c) control group, a) There is a Negative correlation between the levels of IAP and Reticulocyte count in this group $(r=-0.3)$. b) There is a Negative correlation between the levels of IAP and Reticulocyte count in this group ( $r=-0.3)$. c) There is a Positive correlation between the levels of IAP and Reticulocyte count in this group ( $r=0.2)$

infection seen in advancing HIV infection (increasing IAP concentration) causing ineffective erythropiesis. There is also the decreased responsiveness to erythropoietin, blunted erythropoietin production in response to renal damage leading to reduction in reticulocyte count. The control group on the other hand has increasing reticulocyte count with increasing IAP due to the absence of HIV infection and thus a fully functional erythropoietic mechanism to replenish old or destroyed red blood cells. ${ }^{23}$

Furthermore, there was a positive correlation in ART, NART and control groups between percentage neutrophil and IAP 


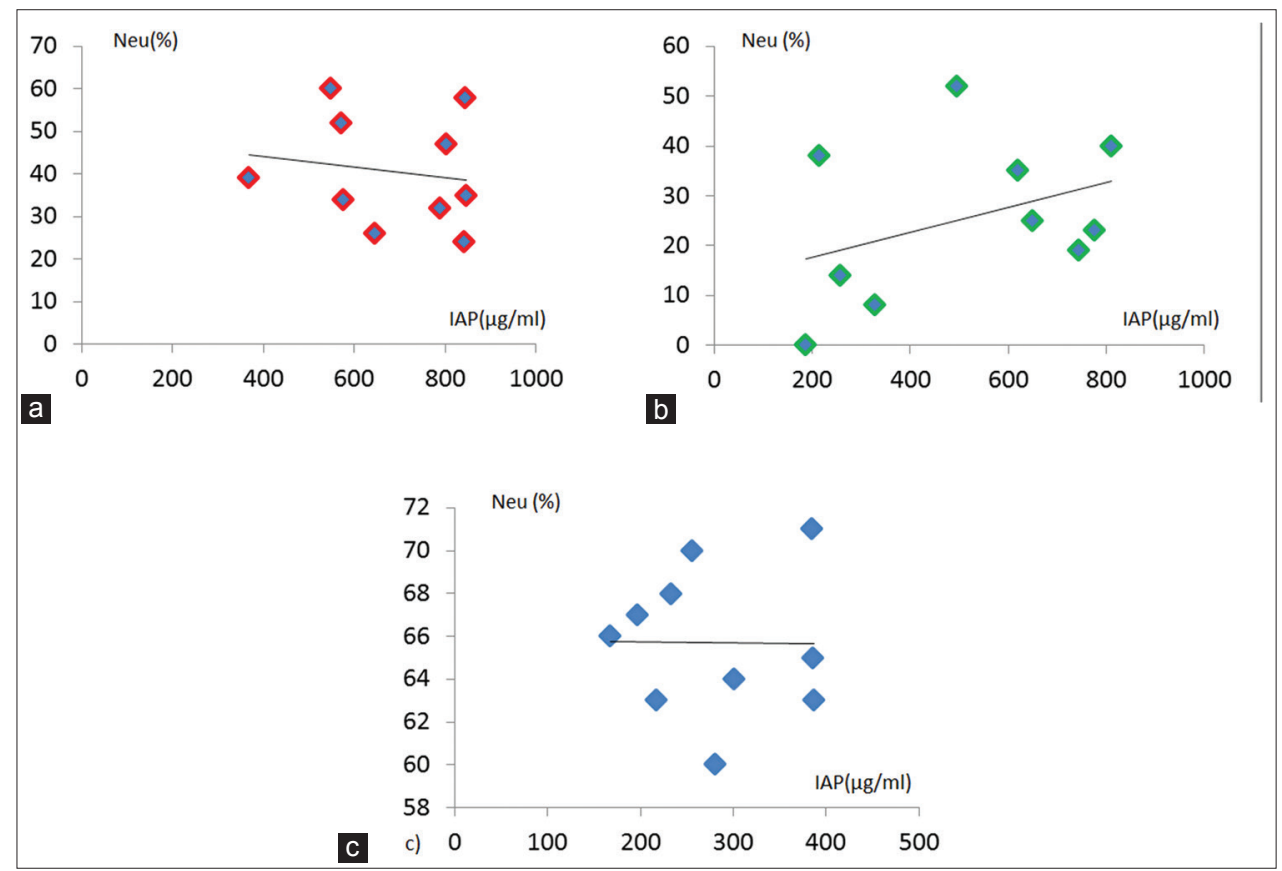

Figure 7: Scatter Plot showing relationship between IAP and percentage Neutrophil in (a) ART (b) NART and (c) control group, (a) There is a Positive correlation between the levels of IAP and Neutrophil in this group ( $r=0.2)$, (b) There is a Positive correlation between the levels of IAP and Neutrophil in this group $(r=0.3)$. (c) There is a Positive correlation between the levels of IAP and Neutrophil count in this group ( $r=0.1)$.

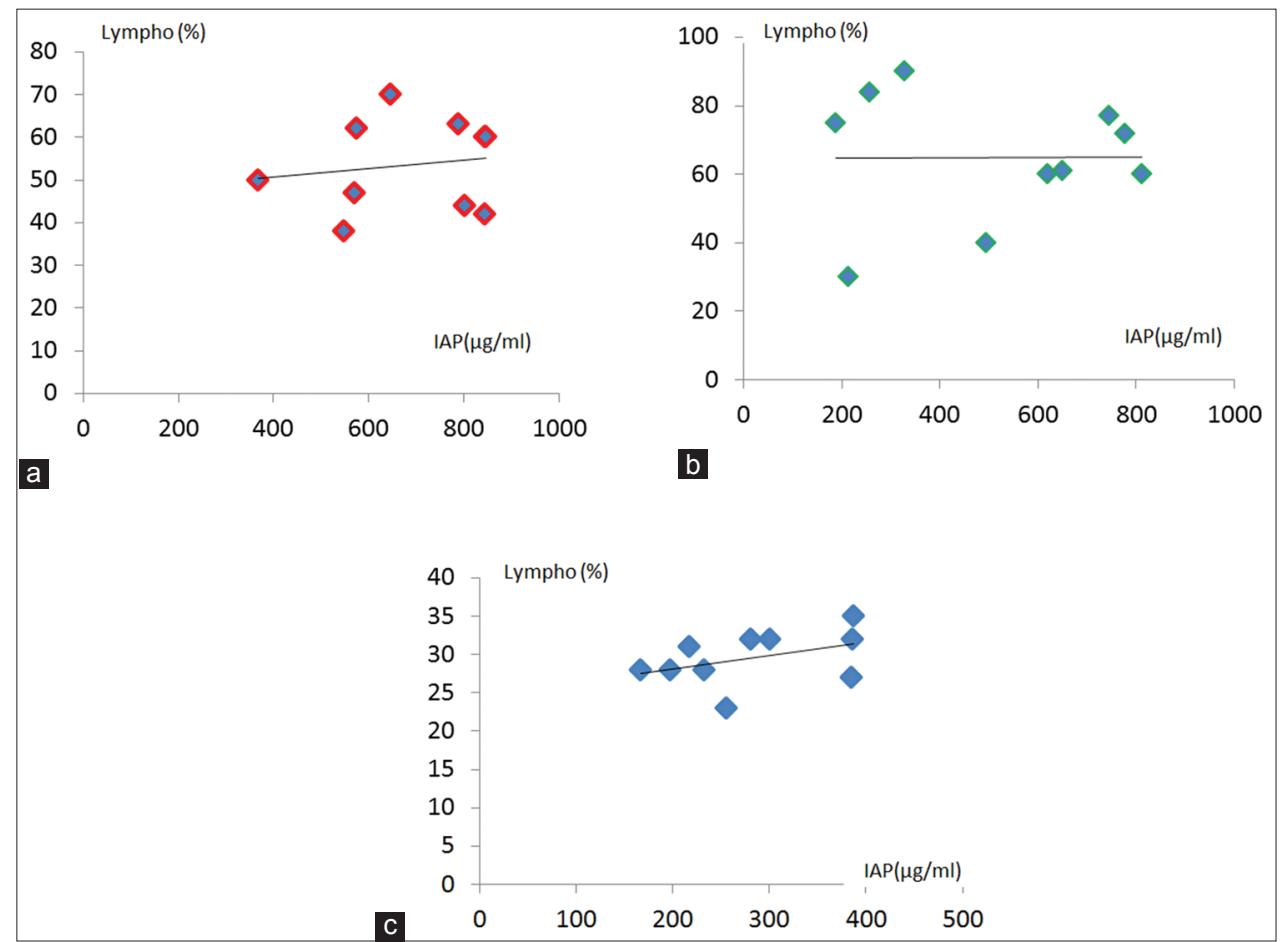

Figure 8: Scatter Plot showing relationship between IAP and percentage Lymphocyte in (a) ART, (b) NART and (c) control group, (a) There is a Negative correlation between the levels of IAP and Lymphocyte in this group $(r=-0.3)$, (b) There is a weak Negative correlation between the levels of IAP and Lymphocyte in this group ( $r=-0.02)$, (c) There is a weak Positive correlation between the levels of IAP and Lymphocyte in this group $(r=0.05)$

concentration as seen in Figure 7. The findings in ART and NART was inconsistent while that of the control group was consistent with that of previous studies ${ }^{24,25}$ possibly due to undetected asymptomatic opportunistic bacterial infections which causes neutrophilia which might be present in participants in this study without showing off symptoms. 


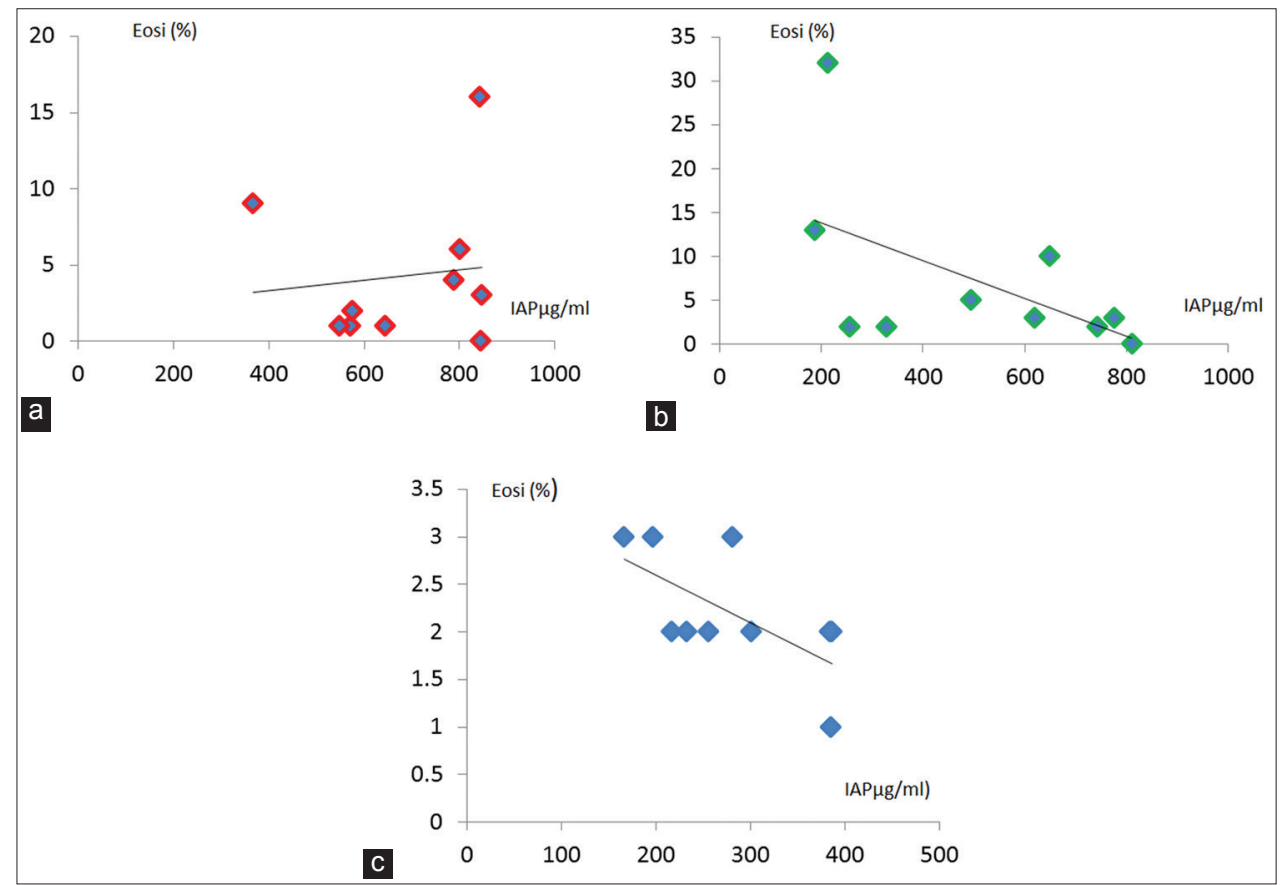

Figure 9: Scatter Plot showing relationship between IAP and percentage Eosinophil in (a) ART (b) NART and (c) control group, a) There is a Positive correlation between the levels of IAP and Eosinophil in this group $(r=0.6)$, b) There is a Negative correlation between the levels of IAP and Eosinophil in this group $(r=-0.4), c)$ There is a Negative correlation between the levels of IAP and Eosinophil in this group ( $r=-0.2)$.

Percentage lymphocyte showed a negative correlation with the levels of IAP in both ART and NART groups and a positive correlation in the control group as shown in Figure 8. This was consistent with the findings of the earlier studies. ${ }^{22,25}$ There is reduced lymphocyte count due to the direct destruction of $\mathrm{CD}^{+}{ }^{+} \mathrm{T}$-Lymphocyte which makes up about $60 \%-70 \%$ of $\mathrm{T}$ - lymphocyte population by HIV viral particles.

It is essential to note that elevation in IAP is not specific for HIV infection as other disease conditions that cause any form of infection, tissue injury or inflammation as seen in most viral, bacterial and parasitic infections will produce an elevated IAP. The CD4 count however is a more specific marker as HIV particles directly infects CD4 T-cells causing eventual destruction of the cell and thus general reduction in its count although some other disease conditions can also produce reduction in CD4 cell count as seen in Tuberculosis.

\section{CONCLUSION}

The finding of this study indicates a strong negative relationship between IAP and other immunohaematological parameters for monitoring Immune status in HIV infection; however the information gotten is not sufficient to indicate IAP as a predictor of immune status in HIV infection. More studies should be done to evaluate IAP concentration at different clinical stages of HIV infection while classifying
HIV infected individuals as short-term progressors, rapid progressors or long-term non-progressors to determine if IAP can be an accurate predictor of immune status in HIV infection.

\section{ACKNOWLEDGEMENTS}

The authors are indebted to the Management and Staff of the HIV Care and Support Center of the Federal Teaching Hospital, Ido, Ekiti State for the administrative approval granted for the use of their facility as study center. Our appreciations also go to all the patients that participated in this study, particularly for their consent and co-operation.

\section{Competing interests}

The authors declare no competing interest.

\section{REFERENCES}

1. Sebastian L. The Pathology of HIV infection. Leprosy Review 2002; 73:64-71.

2. Erhabor O, Uko EK and Adidas T. Absolute lymphocyte count as a marker for CD4 T-Lymphocyte count: criterion for initiating ART in HIV infected Nigerians. Nigerian Journal of Medicine2006; 15(1):56-59.

3. Moses A, Nelson $\mathrm{J}$ and Bagby GC. The influence of Human immunodeficiency virus-1 on hematopoiesis. Blood 1998; 91:1479-1495.

4. CoyleTE. Hematologiccomplications of humanimmunodeficiency virus infection and the acquired immunodeficiency syndrome. Medical Clinical North America 1997; 81: 449-470. 
5. Akele RY, Olayanju AO, Bala ND, Chollom SC, Enitan SS and Irec J. Haematological parameters in Human Immunodeficiency virus positive individuals on Different HAART regimen. World Journal of Pharmaceutical Research 2015; 4(12):10-21.

6. Javier OR, Signed EP, Catalina C, Carlos EP, Jessica TM, Juan B, et al. Predictions of CD4 lymphocytes' count in HIV patients from complete blood count. Bio Medical Physics 2013; 13:3.

7. Ramesh SP. Immunopathogenesis of HIV infection. Indian Journal of Medicine 2005; 121:240-255.

8. Muhammad YD, Rizwan AQ and Naila B. Total lymphocyte Count as a Substitute to CD4 count in Management of HIV individuals in Resource Limited Society. Journal of Ayub Medical College Abbottabad 2015; 27(1):1-4.

9. GrossmanZ, Meier SM, Sousa AE, Victorin RM and Paul WE. CD4+ T cell depletion in HIV infection: Are we close to understanding the cause? National Medicine 2002; 8: 319-323.

10. Yung $C L$, Pun $C Y$ and Sow HK. Immunosuppressive acidic protein in patients with lung cancer. Chest 1992; 102:1491-1495.

11. Thierry F, Najet $\mathrm{MN}$ and Dominique P. Protein Structure and Molecular Enzymology. Biochimica Biophysica Acta 2000; 1482(1): 157-171.

12. Tamura $\mathrm{K}$, Shibata $\mathrm{Y}$, Matsuda $\mathrm{Y}$ and Ishid $\mathrm{N}$. Isolation and Characterization of an Immunosuppressive Acidic Protein from Ascitic Fluids of Cancer Patients. Cancer Research 1981; 41: 3244-3252.

13. Katsuyuki A, Takafunmi $U$, Mashahiro A and Masahiko T. Immunohistochemical determination of Immunosupressive Acidic Protein in Reactive and Neoplastic Diseases of Macrophage.Cancer1987; 60:2424-2427.

14. Festing MF and Altman DG. Guidelines for the design and statistical analysis of experiments using laboratory animals. ILAR J 2002; 43(4):244-258.

15. Mwenda V, Njaguna J and Musa M. Efficacy of Total Leucocyte count as a Surrogate for CD4 cell count in HIV infected adults in the era of higher treatment cut off and less finding: a cross- sectional study. East and Central Africa Medical Journal 2015;2:84-88.

16. Battaglia F, Scambia G, Benedetti $P$ and Mancuso $S$. Immunosuppressive Acidic Protein (IAP) and Squamous Cell Carcinoma Antigen (SCC) in Patients with Cervical Cancer. Gynecologic oncology 1994; 53(2):176-182.

17. Igarashi $\mathrm{T}$, Murakami $\mathrm{S}$, Isaka $\mathrm{S}$, Okano $\mathrm{T}$, Shimazaki $\mathrm{J}$ and Matsuzaki O. Serum immunosuppressive acidic protein as a tumor marker for renal cell carcinoma. European Urology 1991; 19(4):332-335.

18. Matsumoto K, Iwamura M, Muramoto M, Suyama K, Tabata K, Minei $S$, et al. Prognostic value of serum immunosuppressive acidic protein in renal cell carcinoma. Nihon Hinyokika Gakkai Zasshi 2002; 93(4):548-554.

19. Volberding PA, Levine AM and Dieterich D. Anemia in HIV infection: clinical impact and evidence based management strategies. Clinical Infectious Disease 2004; 38:1454-1463.

20. Sujata E, Dinesh S, Raj B and Aryah S. Association of haematological profile of human immunodeficiency Virus positive patients with clinicoimmunologic stages of the disease. Journal of Laboratory Physicians 2013; 5(1):34-37.

21. Mohsen M, Farshid R, Mohammad R and Majid A. Prevalence, severity and related factors of anaemia and HIVIAIDS Patients. Journal of Research in Medical Sciences 2012; 17(2):138-142.

22. Kasthuri A, Sanjeevan S and Kar P. A study of hematological manifestations of HIV infection. Indian Journal of Sexually Transmitted Diseases 2006; 27(1):9-16.

23. Humbleton J. Haematologic complications of HIV infection. Oncology 1996; 10(5):671-680.

24. Mike F, Paul M, Waters A and Smith A. Incidence and mechanism of neutropeania and thrombocytopeania in patients with HIV infection. British Journal of Haematology1987; 66(3):337-340.

25. Gil CS, Denise MB and Alcyone AM. Haematological abnormalities in HIV infected patients. International Journal of Infectious Diseases 2011; 15(12): e808-e811.

\section{Author's contribution:}

OAO - Concept and design of the study, reviewed the literature, prepared first draft of manuscript and critical revision of the manuscript; OTD - Concept, analysis, collected data and helped in preparing first draft of manuscript; ARY - Literature search, statistically analysis and interpretation; ESS - Preparation and critical revision of the manuscript; OA - Supervision, collected data and review of study; SJO - Literature search, approval of manuscript and review of study; OAJ - Literature search, approval of manuscript and review of study.

\section{Orcid ID:}

Dr. Olayanju AO: http://orcid.org/0000-0001-9365-5564

Dr. Oyebola TD: http://orcid.org/0000-0003-0251-6310

Dr. Akele RY: http://orcid.org/0000-0003-2006-0820

Source of funding: Nil, Conflict of interest: None. 